\title{
Modelling the Kinetics of Jatropha Oil Transesterification
}

\author{
Aldo A. Okullo' ${ }^{1}$ Abraham K. Temu ${ }^{2}$ \\ ${ }^{1}$ Department of Chemistry, Faculty of Science, Kyambogo University (KYU), Kampala, Uganda \\ ${ }^{2}$ Department of Chemical and Mining Engineering, University of Dar Es Salam (UDSM), \\ Dar Es Salaam, Tanzania \\ Email: okulloapita@yahoo.com, Atemu8@yahoo.com
}

Received 10 October 2014; accepted 8 April 2015; published 10 April 2015

Copyright (C) 2015 by authors and Scientific Research Publishing Inc.

This work is licensed under the Creative Commons Attribution International License (CC BY). http://creativecommons.org/licenses/by/4.0/

(c) (i)

\section{Abstract}

Kinetics of a chemical reaction provides an important means of determining the extent of the reaction and in reactor designs. Transesterification of jatropha oil with methanol and sodium hydroxide as a catalyst was conducted in a well mixed reactor at different agitation speeds between 600 and $800 \mathrm{rpm}$ and temperature range between $35^{\circ} \mathrm{C}$ and $65^{\circ} \mathrm{C}$. The effect of variation of temperature and mixing intensity on rate constants were studied. The initial mass transfer controlled stage was considered negligible using the above impeller speeds and second order mechanism was considered for the chemically controlled kinetic stage. Samples were collected from the reaction mixture at specified time intervals and quenched in a mixture of tetrahydrofuran (THF) and sulphuric acid. The mixture was centrifuged at $2000 \mathrm{rpm}$ for 15 minutes and the methyl ester was separated from the glycerol. The ester was washed with warm water $\left(50^{\circ} \mathrm{C}\right)$, dried and analysed using gas chromatography coupled with flame ionization detector (GC/FID) to determine free and total glycerine and methyl ester. A mathematical model was fitted using second order rate law. High temperature and high mixing intensity increased reaction rates. The model fitted well with a high correlation coefficient $\left(R^{2}\right)$ of 0.999 .

\section{Keywords}

Second Order Kinetics, Rate Constants, Jatropha Oil, Transesterification

\section{Introduction}

Biodiesel is produced from plant oils and animal fats. Production of biodiesel has become widespread because 
of its reliability and potential energy sustainability. Efforts are being made to initiate and encourage biodiesel production at community levels in many developing countries [1]. In Tanzania, jatropha curcus growing is being encouraged among local communities in marginal lands by Tanzania Traditional Energy Development Organisation (TaTEDO) and "Kampuni ya Kusambaza Teknolojia" (Kakute) [2]. Oils derived from these sources are utilized locally for lighting, in generators, grain milling and soap making. However, there are several drawbacks in these initiatives; for example, lack of knowledge on the characteristics of feedstock and their development, high cost of inputs for cultivation and processing could hamper the process. In addition, machineries and equipment used in cultivation are not locally obtainable including those needed for de-husking, extraction, refining and processing.

To design a suitable reactor for biodiesel production, kinetic mechanisms and reaction rate constants have to be known. Kinetic study of palm oil transesterification in a batch reactor using potassium hydroxide as a catalyst showed the conversion of triglyceride, diglyceride and monoglyceride as being second-order [3] [4]. On the other hand, [5] [6] investigated the kinetics of sunflower and brassica carinata oils methanolysis and reported initial mass transfer controlled kinetics followed by second-order kinetically controlled rates. A research on the kinetics of transesterification of soybean oil with methanol using a shunt reaction reported that mass transfer control reaction is negligible with the stirring speed of $600 \mathrm{rpm}$ and the kinetically controlled chemical reaction was second-order [7]. A study on the kinetics of hydroxide-catalysed methanolysis of crude sunflower oil for the production of fuel-grade methyl esters reported the mass transfer region to be effectively minimized using agitation speeds between 400 and $600 \mathrm{rpm}$ [8]. A kinetic study on the methanolysis of sunflower oil at low temperatures using simplified rate equations reported a sigmoidal kinetic where mass transfer initially control the kinetic followed by a chemically controlled kinetic region [9]. However, data on the kinetics of jatropha oil transesterification is still very scanty. In addition, fatty acid compositions vary from plant to plant and depend on geographical location, altitude and other environmental factors.

This study was conducted to determine the effect of temperature and mixing intensity on the rate constants and therefore the conversion of jatropha oil using second order rate law. A mathematical model according to [5] [6] [10] was used as we considered it to be rigorous. This would provide information that could be used in reactor designs for jatropha biodiesel production. Jatropha plant was chosen for the study because of its high oil content. It is also non-edible and grows well in the tropical environment.

\section{Kinetic Mathematical Model}

Triglycerides make up the major components of plant and animal oils. Its transesterification is consecutive and reversible, represented by the equations according to [4]-[6] [11]-[13].

$$
\begin{gathered}
\mathrm{TG}+\mathrm{OH} \underset{k_{2}}{\stackrel{k_{1}}{\Leftrightarrow}} \mathrm{DG}+\mathrm{ME} \\
\mathrm{DG}+\mathrm{OH} \underset{k_{4}}{\stackrel{k_{3}}{\Leftrightarrow} \mathrm{MG}+\mathrm{ME}} \\
\mathrm{MG}+\mathrm{OH} \underset{k_{6}}{\stackrel{k_{5}}{\Leftrightarrow} \mathrm{GL}+\mathrm{ME}}
\end{gathered}
$$

where $k_{1}, k_{3}$ and $k_{5}$ are rate constants for forward reactions; $k_{2}, k_{4}$ and $k_{6}$ are rate constants for reverse reactions, $\mathrm{OH}=$ alcohol, $\mathrm{ME}_{1}, \mathrm{ME}_{2}$ and $\mathrm{ME}_{2}=$ fatty acid methyl esters, $\mathrm{TG}=$ triglyceride, $\mathrm{DG}=$ diglyceride, $\mathrm{MG}=$ monoglyceride and $\mathrm{GL}=$ glycerol .

The consecutive reactions can be forward reactions, reversible or equilibrium depending on several working conditions including the choice of catalyst and molar ratio of alcohol to oil. Oil and methanol are not miscible, the reaction system forms two phases at the initial stage therefore mass transfer controls the kinetic at this stage. However, using the impeller speed of 600 to $800 \mathrm{rpm}$ the initial mass transfer stage was considered negligible and chemical reaction controlled the kinetics. The differential kinetic rate law equations developed from Equations (1) to (3) are given in Equations (4) to (9) according to [10] [12] [13]. This system of equations can be resolved using numerical methods: 


$$
\begin{aligned}
\frac{\partial \mathrm{TG}}{\partial t} & =-k_{1}[\mathrm{TG}][\mathrm{OH}]+k_{2}[\mathrm{DG}][\mathrm{ME}] \\
\frac{\partial \mathrm{DG}}{\partial t} & =k_{1}[\mathrm{TG}][\mathrm{OH}]-k_{2}[\mathrm{DG}][\mathrm{ME}]-k_{3}[\mathrm{DG}][\mathrm{OH}]+k_{4}[\mathrm{MG}][\mathrm{ME}] \\
\frac{\partial \mathrm{MG}}{\partial t} & =k_{3}[\mathrm{DG}][\mathrm{OH}]-k_{4}[\mathrm{MG}][\mathrm{ME}]+k_{5}[\mathrm{MG}][\mathrm{OH}]-k_{6}[\mathrm{GL}][\mathrm{ME}] \\
\frac{\partial[\mathrm{GL}]}{\partial t} & =k_{5}[\mathrm{MG}][\mathrm{OH}]-k_{6}[\mathrm{GL}][\mathrm{ME}] \\
\frac{\partial[\mathrm{ME}]}{\partial t} & =k_{1}[\mathrm{TG}][\mathrm{OH}]-k_{2}[\mathrm{DG}][\mathrm{ME}]+k_{3}[\mathrm{DG}][\mathrm{OH}] \\
& -k_{4}[\mathrm{MG}][\mathrm{ME}]+k_{5}[\mathrm{MG}][\mathrm{OH}]-k_{6}[\mathrm{GL}][\mathrm{ME}] \\
\frac{\partial[\mathrm{OH}]}{\partial t} & =-\frac{\partial[\mathrm{ME}]}{\partial t}
\end{aligned}
$$

where [TG], [DG] [MG], [GL], [OH], and [ME] are mole concentrations of triglycerides, diglyceride, monoglyceride, glycerine, alcohol, and methyl esters respectively in the reaction mixture (mol/L).

Differentiation of the mole concentrations with respect to time on the left hand side of Equations (4)-(9) were estimated from the slopes of experimental concentration-time curves at various reaction times using three-point method. The second order differential system of equations was transformed appropriately and resolved using least-squares regression technique with Matlab computer Software, Version 7.10.0.499 (R2010a), the Mathworks, USA. The Equations (4)-(9) gave a system of six nonlinear equations with six unknowns after rearrangement (Equation (10)):

$$
\left[\begin{array}{cccccc}
-a_{11} & a_{12} & 0 & 0 & a_{5} & -a_{16} \\
a_{21} & -2 a_{22} & a_{23} & 0 & 0 & 0 \\
0 & a_{32} & -2 a_{33} & -a_{34} & 0 & 0 \\
0 & 0 & a_{43} & -a_{44} & -a_{45} & a_{46} \\
a_{51} & 0 & 0 & -a_{54} & -3 a_{55} & -a_{56} \\
-a_{61} & 0 & 0 & a_{64} & 3 a_{65} & a_{66}
\end{array}\right]\left[\begin{array}{l}
k_{1} \\
k_{2} \\
k_{3} \\
k_{4} \\
k_{5} \\
k_{6}
\end{array}\right]=\left[\begin{array}{c}
\frac{\partial \mathrm{TG}}{\partial t} \\
\frac{\partial \mathrm{DG}}{\partial t} \\
\frac{\partial \mathrm{MG}}{\partial t} \\
\frac{\partial \mathrm{GL}}{\partial t} \\
\frac{\partial \mathrm{ME}}{\partial t} \\
\frac{\partial \mathrm{OH}}{\partial t}
\end{array}\right]
$$

where $a_{11}-a_{66}$ are measured data points (concentrations); the dependent variables are the differentiations of mole concentrations on the right hand side of Equation (10) and $k_{1}, k_{2}, k_{3}, k_{4}, k_{5}$ and $k_{6}$ are rate constants. The methodology followed to determine the effective rate constants is represented by the flow chart in Figure 1. This method was suggested by [14] Lamina et al., (2010) where instead of using rate constant values from literature as initial guesses, the slopes from concentration-time data are used as initial values of rate constants to start the optimisation.

\section{Materials and Methods}

\subsection{Materials}

Jatropha seeds were obtained from Arusha in Tanzania. Oil extraction was done using a Screw oil pressing ma- 


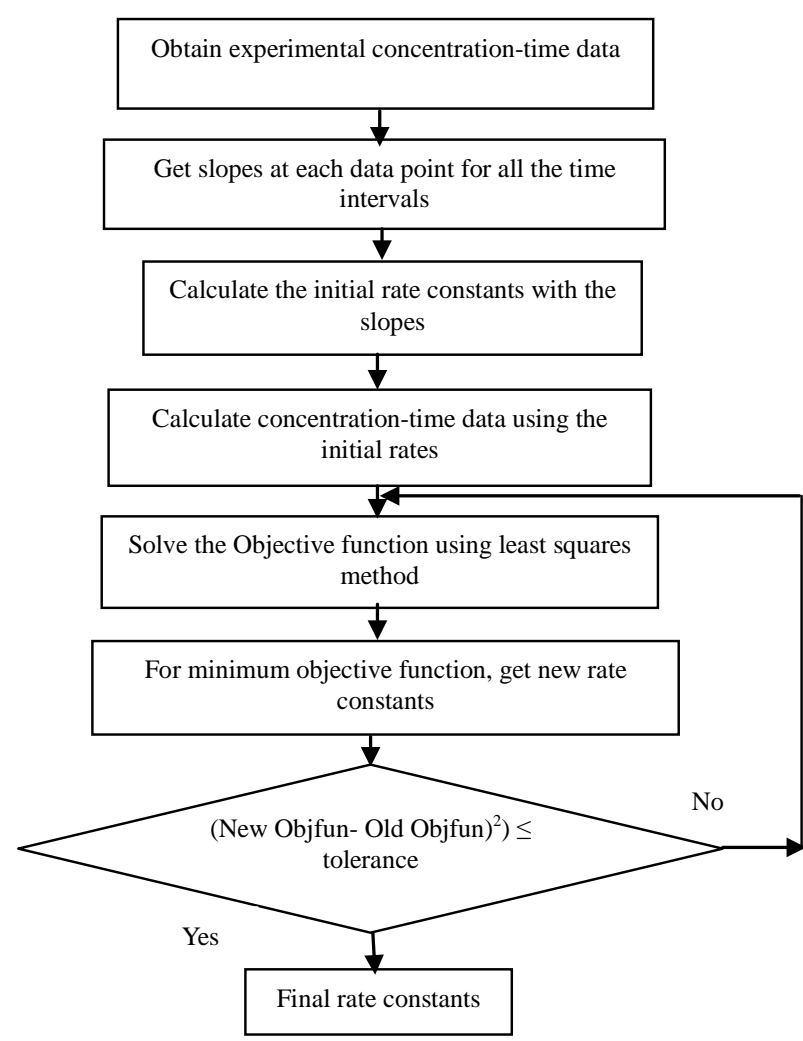

Figure 1. Flow chart for the determination of rate constants (adopted from [14]).

chine, model 6YL-95A, China. It was water degummed by heating it between $70^{\circ} \mathrm{C}$ to $80^{\circ} \mathrm{C}$ for 15 minutes while stirring before neutralizing with sodium hydroxide to reduce free fatty acids (FFA) content. Gas chromatography grade chemicals and biodiesel standards were obtained from Supelco Sigma Aldrich, Germany and Choice Analytical Pty Ltd., Thornig, Australia.

\subsection{Methods}

Free fatty acid of the degummed oil was determined using titration methods as described in ASTM D 664 [15]. On the basis of the FFA value; a calculated strength of sodium hydroxide solution was prepared to neutralize the oil. The alkali solution was added to the oil at room temperature $\left(30^{\circ} \mathrm{C}\right)$ while stirring at $250 \mathrm{rpm}$ using a stirrer Heidolph RZR 50 L, Germany and the reaction was allowed to continue for 10 minutes after which the mixture was heated to $70^{\circ} \mathrm{C}$ to break the soap formed. This was followed by separation using a Martin Christ centrifuge (UJ3, Germany) run at $4500 \mathrm{rpm}$ for 20 minutes. The oil was washed three times with warm water $\left(50^{\circ} \mathrm{C}\right)$ and dried using a Heidolph Laborota (4001, Germany rotovapour) and it was ready for use.

\subsection{Procedure}

The reaction was carried out in a $500 \mathrm{ml}$ round bottom flask as a reactor fitted with a reflux condenser, a mechanical stirrer, a thermometer and a sampling port. Oil was pre-heated at $110^{\circ} \mathrm{C}$ for 30 minutes to drive off moisture before it was put into the reactor. Sodium hydroxide, (0.5\% wt of oil) was dissolved in Methanol (molar ratio, 6:1 of oil) when the oil was drying and the mixture transferred into the reactor while stirring. The Experiment was set following the optimized conditions according to [16]; temperature range $\left(35^{\circ} \mathrm{C}\right.$ to $\left.65^{\circ} \mathrm{C}\right)$, stirring rate (600 to $800 \mathrm{rpm})$, and the reaction proceeded for 120 minutes.

\subsection{Sample Preparations and Analysis}

Samples of $5 \mathrm{~mL}$ were drawn after every 5 minutes initially and placed in test tubes containing a mixture of 1 
$\mathrm{mL}$ tetrahydrofuran (THF) and $1 \mathrm{~mL}$ of $0.5 \mathrm{M}$ sulphuric acid. The THF was used to dilute the system and stop the reaction while the acid was to neutralize the catalyst. A previous study indicated that this treatment stops the reaction effectively [17]. After thirty minutes, the sampling frequency was changed to 30 minutes intervals. The sample was transferred into a centrifuge and run at $2000 \mathrm{rpm}$ for 15 minutes to separate the ester from the glycerol. The extracted ester was washed three times with warm water $\left(50^{\circ} \mathrm{C}\right)$, dried using anhydrous sodium sulphate, filtered and kept below $-2^{\circ} \mathrm{C}$ till analysis. Samples were prepared and derivatized according to [15] method before injection into the GC. Gas Chromatography equipment Shimadzu 2010 (model A17, AAF V3, Japan) was used for the determination of free, bound and total glycerines.

\subsection{Simulation of the Kinetics}

After obtaining the experimental concentration-time data, the slopes of the curves were determined using cubic spline method (MATLAB). These slopes acted as initial guesses for rate constants and the optimisation procedure started using least squares.

Optimisation of the rate constants was carried out using a Matlab optimisation solver fsolve-medium scale Levenberg-Marquardt which minimized the objective function of Equation (11) hence giving values of the rate constants.

$$
E^{2}=\sum_{i=1}^{n}\left[b_{i}-\left(a_{i 1} k_{1}+a_{i 2} k_{2}+a_{i 3} k_{3}+\cdots+a_{n 6} k_{6}\right)\right]^{2}
$$

\section{Results and Discussions}

Triglycerides (TG) were rapidly converted to diglyceride (DG) with a simultaneous rise in methyl ester (ME) within ten to fifteen minutes (Figure 2). Diglyceride, on the other hand was converted to monoglyceride (MG) and monoglyceride to glycerine (GL). After 20 minutes, the reaction slowed down and all TG, DG, MG and ME levelled up as the reaction tended towards equilibrium. Methyl ester conversion was $96 \% \mathrm{wt}$ for a temperature of $55^{\circ} \mathrm{C}$ for a reaction of two hours. The residual TG was $1.7 \%$, DG, $2.5 \%$ and MG, $0.07 \%$ (Figure 2). The reaction had not reached completion after two hours. Reasons could be due to low catalyst loading $(0.5 \%$ wt of oil) as opposed to the recommended literature value of $1 \%$ (wt of oil) [5] [7] [8].

Methanol loss through evaporation during transesterification could have been another source of problem for incomplete conversion. It is interesting to note that methyl ester conversion was high even at the initial stage of 5 minutes (73\%) and there was no time lag at this stage that was reported in [5]-[8] [10] [11]. This can be explained by the fact that high mixing intensity used $(600 \mathrm{rpm})$ and high temperature $\left(55^{\circ} \mathrm{C}\right)$ produced homogeneous oil-methanol mixture where mass transfer control was negligible. This observation was also reported by Noureddini and Zhu and Bambase et al. [7] [8].

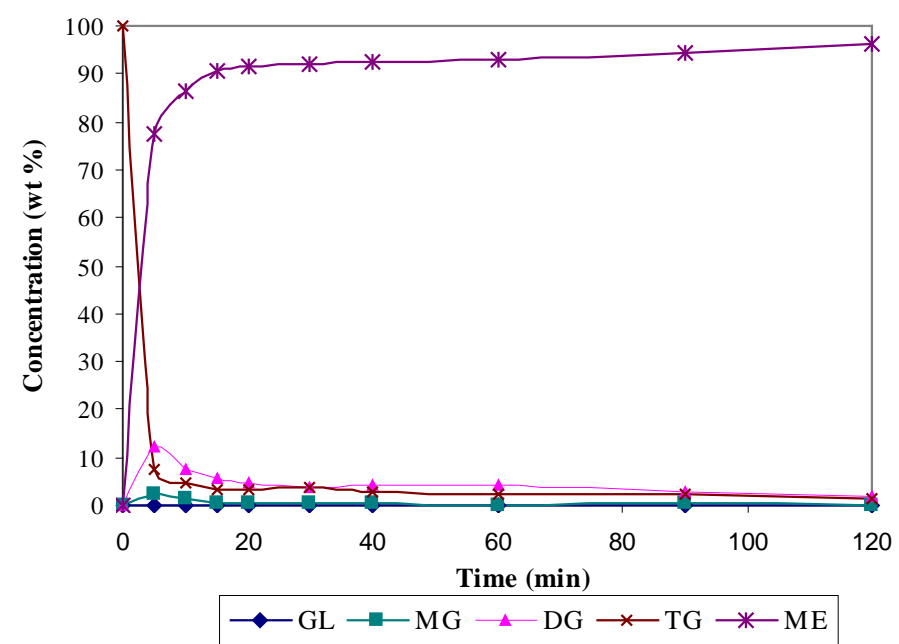

Figure 2. Concentration of reaction mixture at $55^{\circ} \mathrm{C}$; stirring rate $600 \mathrm{rpm}$; molar ratio $6: 1$ and catalyst loading of $0.5 \%$ wt of oil. 
Figure 3 shows the data fit of methyl ester purity (\%). The high value of correlation coefficient $\left(\mathrm{R}^{2}=0.999\right)$ indicating perfect goodness of fit.

\subsection{Effect of Temperature}

Figure 4 shows the effect of temperature on the conversion of jatropha oil to biodiesel. Increasing temperature increases conversion. This is in line with the kinetic molecular theory of matter which states that matter is composed of atoms and molecules which are always in constant motion. When there is an increase in temperature, the atoms and molecules gain kinetic energy and move faster and more collisions result that may cause breaking of bonds. Here again it can be seen that there was no delay in methyl ester appearance even at a low temperature $\left(35^{\circ} \mathrm{C}\right)$ confirming that mass transfer controlled stage was unimportant in this reaction due to high mixing intensity. Conversions were superior even at equilibrium for high temperatures of $45^{\circ} \mathrm{C}, 55^{\circ} \mathrm{C}$ and $65^{\circ} \mathrm{C}(94 \%, 96 \%$ and 98\% respectively) (Figure 4). Methanol becomes more soluble in oil at higher temperatures.

\subsection{Effect of Stirring}

Effect of mixing intensity (stirring) on the conversion of jatropha oil is depicted in Figure 5. High stirring speed

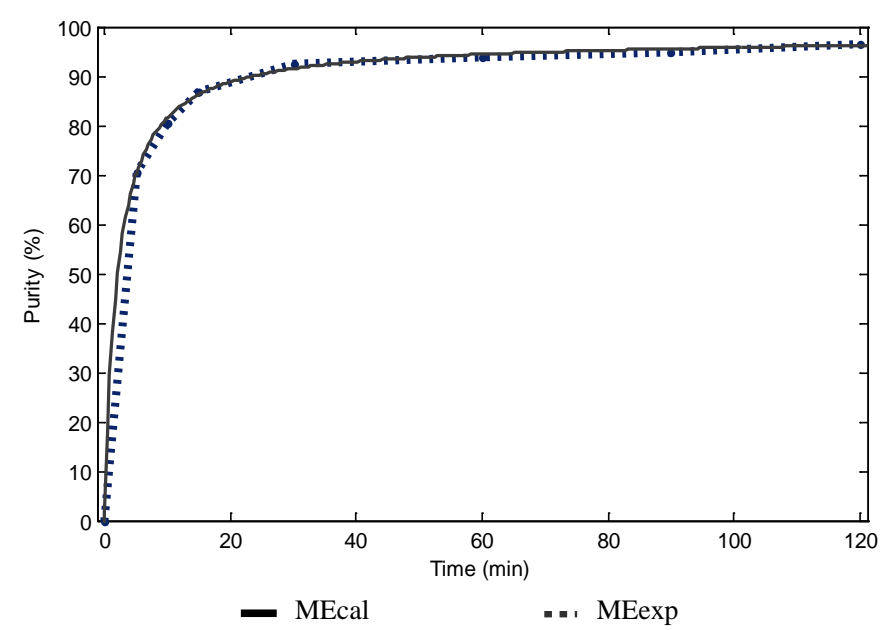

Figure 3. Methyl ester purity (\%) at reaction temperature of $55^{\circ} \mathrm{C}$, stirring rate; $600 \mathrm{rpm}$.

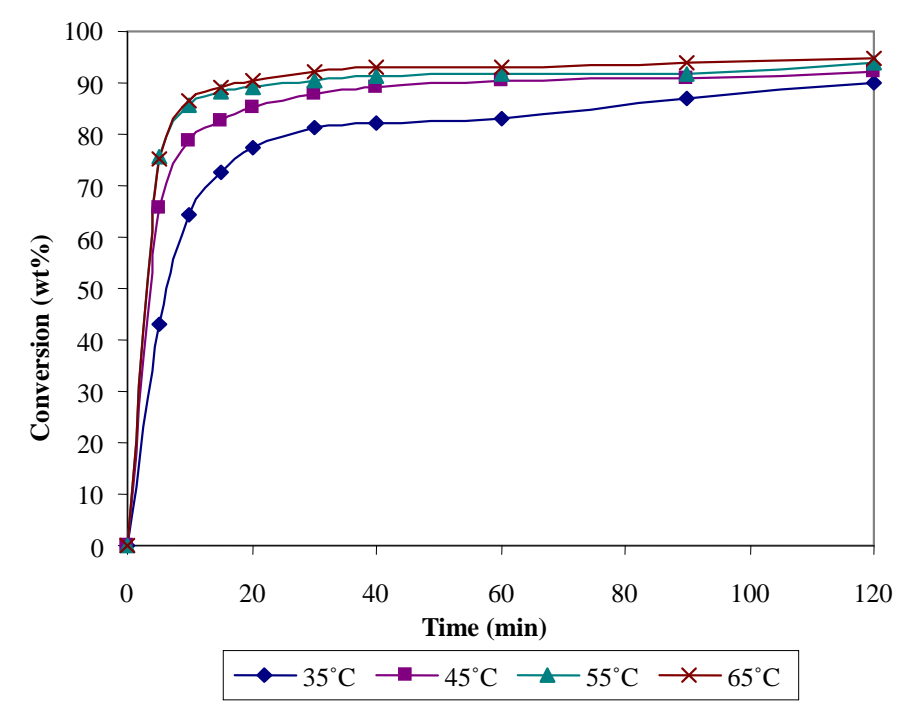

Figure 4. Fatty methyl ester conversion (wt\%) at different temperatures. 


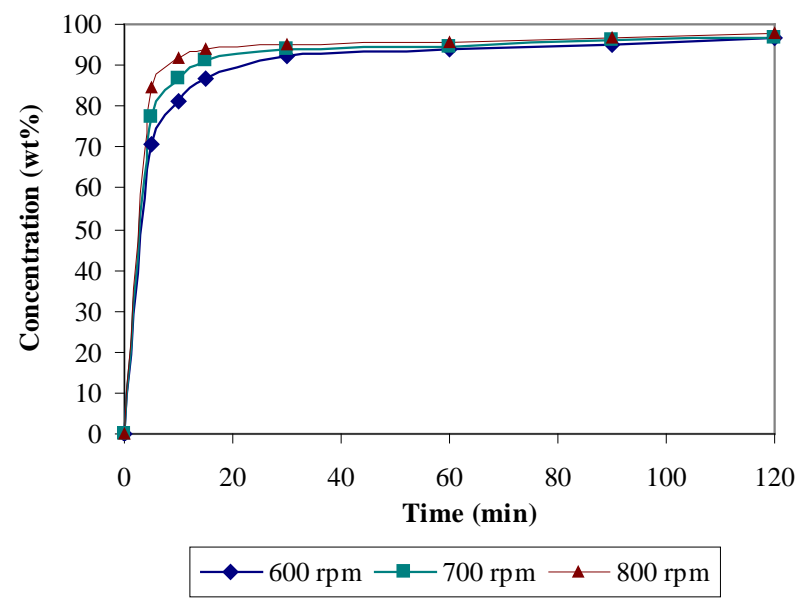

Figure 5. Fatty methyl ester conversion (wt\%) at different stirring speeds.

(600, 700 and $800 \mathrm{rpm}$ ) favoured conversion. The intermolecular forces are broken down by the intensity of mixing making the oil-methanol solution homogeneous without phase formation. Noureddini and Zhu [7] reported that at stirring speed of $600 \mathrm{rpm}$, the mass transfer controlled reaction is eliminated while [8] reported the value of $400 \mathrm{rpm}$. It can be observed that after 30 minutes of the reaction, increasing mixing intensity becomes unnecessary because the methyl ester produced is soluble in oil and in methanol and it acts as a co-solvent where the system is homogeneous and only chemical reaction controls the kinetic (Figure 5).

\subsection{Activation Energy and Rate Constants}

Even at a low temperature $\left(35^{\circ} \mathrm{C}\right)$, the rate constant values for the first forward reactions were high, implying that there was no resistance by the mass transfer at the initial stage. When the temperature increased, rate constant values increased, the rate determining step was for the reversible reaction DG TG. Considering forward reactions alone, the reaction DG to MG $\left(k_{3}\right)$ is slower than those of TG to DG $\left(k_{1}\right)$ and MG to GL $\left(k_{5}\right)$. This explains why there is a slight accumulation of DG in the reaction mixture (as was seen in Figure 2). High concentrations of TG and DG did offset the reversible reaction effects even at high temperatures (Table 1).

To study the temperature dependence on rate constants, Arrhenius equation was used. $\mathrm{It}$ is expressed as lnk $=$ $\ln \mathrm{A}-\mathrm{Ea} / \mathrm{RT}$. Where $\mathrm{A}=$ pre-exponent factor; $\mathrm{Ea}=$ activation energy; $\mathrm{R}=$ universal gas constant $(\mathrm{J} / \mathrm{mol} \mathrm{K})$ and $\mathrm{T}$ absolute temperature (K). Figure 6 shows the plot of lnk vs 1/T. The slopes give the activation energies (Ea) and the intercepts the pre-exponential factors. As seen from Table 1, forward reactions for the first two reaction steps (TG to DG and DG to MG) have lower activation energies than their reverse counterparts which theoretically would mean more favourable reverse reactions. However, high concentrations of TG and DG and chemically controlled kinetics favour forward reactions, especially at high temperatures.

\section{Conclusion}

High temperature and high mixing intensity increased rate constants and eliminated the initial mass transfer controlled stage. After 30 minutes of reaction, increasing mixing intensity became unnecessary. The methyl ester produced acted as a co-solvent of oil-methanol mixture forming a homogeneous mixture that increased rate constants. Kinetically controlled reaction dominated the transesterification and forward reaction kinetics appeared to be most important. Activation energies obtained in this work compare well with those from literature [7] [11]. It can be concluded therefore that jatropha oil methanolysis follows second order rates. This procedure can be used to predict fatty acid methyl ester conversion.

\section{Acknowledgements}

We acknowledge the supports given by Kyambogo University and Alternative Energy for Sustainable Devel- 
Table 1. Rate constant values.

\begin{tabular}{|c|c|c|c|c|c|c|}
\hline \multicolumn{3}{|c|}{$\mathrm{TG} \rightarrow \mathrm{DG}$} & $\mathrm{DG} \rightarrow \mathrm{MG}$ & \multicolumn{3}{|c|}{$\mathrm{MG} \rightarrow \mathrm{GL}$} \\
\hline $\mathrm{T}\left({ }^{\circ} \mathrm{C}\right)$ & $\mathrm{k}_{1}\left(\mathrm{~L} \cdot \mathrm{mol}^{-1} \cdot \mathrm{min}^{-1}\right)$ & $\mathrm{K}_{2}\left(\mathrm{~L} \cdot \mathrm{mol}^{-1} \cdot \min ^{-1}\right)$ & $\mathrm{K}_{3}\left(\mathrm{~L} \cdot \mathrm{mol}^{-1} \cdot \mathrm{min}^{-1}\right)$ & $\mathrm{K}_{4}\left(\mathrm{~L} \cdot \mathrm{mol}^{-1} \cdot \mathrm{min}^{-1}\right)$ & $\mathrm{K}_{5}\left(\mathrm{~L} \cdot \mathrm{mol}^{-1} \cdot \mathrm{min}^{-1}\right)$ & $\mathrm{K}_{6}\left(\mathrm{~L} \cdot \mathrm{mol}^{-1} \cdot \mathrm{min}^{-1}\right)$ \\
\hline 35 & 0.057 & 0.003 & 0.009 & 0.005 & 0.025 & 0.008 \\
\hline 45 & 0.095 & 0.009 & 0.021 & 0.017 & 0.046 & 0.019 \\
\hline 55 & 0.182 & 0.021 & 0.068 & 0.065 & 0.098 & 0.044 \\
\hline 65 & 0.382 & 0.053 & 0.198 & 0.295 & 0.232 & 0.088 \\
\hline $\mathrm{Ea}\left(\mathrm{kJ} \cdot \mathrm{mol}^{-1}\right)$ & 54.86 & 81.94 & 90.26 & 98.68 & 64.54 & 71.82 \\
\hline $\mathrm{R} 2$ & 0.991 & 0.999 & 0.995 & 0.998 & 0.990 & 0.995 \\
\hline
\end{tabular}

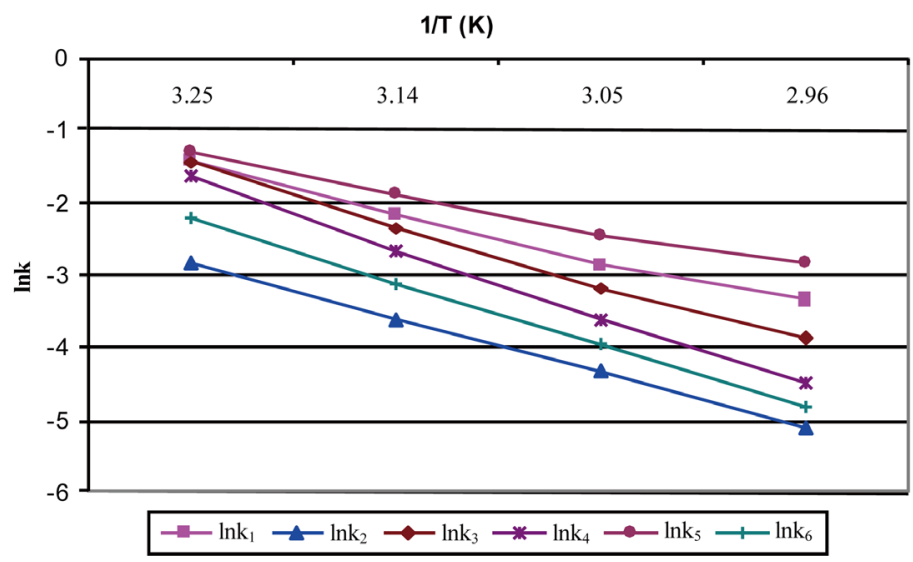

Figure 6. Plot of lnk vs 1/T (K).

opment, Environmental Protection and Poverty Reduction in Tanzania (ESEPRIT) Project which enabled us realise this work.

\section{References}

[1] Keith, O. (2000) A Review of Jatropha Curcas: An Oil Plant of Unfulfilled Promise. Biomass and Bioenergy, 19, 1-15. http://dx.doi.org/10.1016/S0961-9534(00)00019-2

[2] Sulle, E. and Nelson, F. (2009) Biofuels, Land Access and Rural Livelihoods in Tanzania. IIED, London.

[3] Darnoko, D. and Cheryan, M. (2000) Kinetics of Palm Oil Transesterification in a Batch Reactor. Journal of American Oil Chemical Society, 77, 1263-1267. http://dx.doi.org/10.1007/s11746-000-0198-y

[4] Theerayut, L. (2006) Wisutmethangoon Worawut, Prateepchaikul Gumpon, Tongurai Charktir and Allen Michael, Transesterification of Palm Oil in Series of Continuous Stirred Tank Reactor. Asian Journal of Energy and Environment, 7, 336-346.

[5] Vicente, G., Martinez, M., Aracil, J. and Estaban, A. (2005) Kinetics of Sunflower Oil Methanolysis. Industrial Engineering Chemistry Research, 44, 5447-5454.

[6] Vicente, G., Martinez, M. and Aracil, J. (2006) Kinetics of Brassica carinata Oil Methanolysis. Energy and Fuels, 20, 1722-1726. http://dx.doi.org/10.1021/ef060047r

[7] Noureddini, H. and Zhu, D. (1997) Kinetics of Transesterification of Soybean Oil. Journal of American Oil Chemical Society, 74, 1457-1462.

[8] Bambase Manolito Jr., E., Nakamura, N., Tanaka, J. and Matsumura, M. (2007) Kinetics of Hydroxide-Catalyzed Methanolysis of Crude Sunflower Oil for the Production of Fuel-Grade Methyl Esters. Journal of Chemical Technology and Biotechnology, 82, 273-280. http://dx.doi.org/10.1002/jctb.1666

[9] Stamenkovic, O.S., Todorovic, Z.B., Lazic, M.L., Veljkovic, V.B. and Skala, D.U. (2008) Kinetics of Sunflower Methanolysis at Low Temperatures. Bioresource Technology, 99, 1131-1140.

http://dx.doi.org/10.1016/j.biortech.2007.02.028 
[10] Leevijit, T., Worawut, W., Gumpon, P., Charktir, T. and Allen, M. (2004) Second Order Kinetics of Palm Oil Transesterification. The Joint International Conference on Sustainable Energy and Environment (SEE), Hua Hin, 1-3 December 2004.

[11] Freedman Bernard, H., Butterfield Royden, O. and Pryde Everett, H. (1986) Transesterification Kinetics of Soybean Oil. Journal of American Oil Chemical Society, 63, 1375-1380.

[12] Bruno, W., Maicon, T., Apreciado, M. and Alexander, K. (2006) Modelling Chemical Kinetics of Soybean Oil Transesterification Process for Biodiesel Production: An Analysis of Molar Ratio between Alcohol and Soybean Oil Temperature Changes on the Process Conversion Rate. Bioautomation, 5, 13-22.

[13] Karel, K., Frantisek, S., Radek, S. and Jaroslav, M. (2002) Kinetics and Mechanism of the KOH-Catalyzed Methanolysis of Rapeseed Oil for Biodiesel Production. European Journal of Lipid Science Technology, 104, 728-737.

[14] Abdel-Latiff, A.S. and Lamina, A.M. (2010) Determination of the Rate Constants for Consecutive Second Order Irreversible Chemical Reaction Using MATLAB Toolbox. European Journal of Scientific Research, 4, 412-419.

[15] ASTM International (2009) ASTM D 6751-09 Standard Specification for Biodiesel Fuel (B100) Blend Stock for Distillate Fuels.

[16] Okullo, A., Temu, A.K., Ntalikwa, J.W. and Ogwok, P. (2010) Optimization of Biodiesel Production from Jatropha Oil. International Journal of Engineering Research in Africa, 3, 62-74.

[17] Tobias, K. (2004) Kinetic Investigation of Base-Catalyzed Glycerolysis of Fatty Acid Methyl Esters. Doctor of Engineering Dissertation, Faculty of Mathematics and Natural Sciences, Technical University of Berlin Germany, November. 